\title{
Determinants of recent HIV testing among male sex workers and other men who have sex with men in Shenzhen, China: a cross-sectional study
}

\author{
Rui Cai, Jin Zhao, Wende Cai, Lin Chen, Jan Hendrik Richardus, Sake J de Vlas \\ From Abstracts from International Symposium HIV and Emerging Infectious Diseases 2014 \\ Marseille, France. 21-23 May 2013
}

\section{Background}

Men who have sex with men (MSM), including male sex workers (also known as money boys, MBs), are highly vulnerable to HIV, but HIV testing rates remain unacceptably low in China. We compared and separately evaluated determinants of recent HIV testing among MBs and other MSM.

\section{Methods}

We recruited 510 MBs and 533 other MSM in Shenzhen by time-location sampling. We compared their HIV testing history in the last year, socio-demographics and behavioral information, and we performed logistic regression to identify determinants of having been tested in the last year among MBs and other MSM.

\section{Results}

Compared to other MSM, MBs had a slightly lower rate of HIV testing (43\% versus $48 \%, \mathrm{p}=0.10$ ). MBs having multiple anal sex partners (adjusted odds ratio, AOR $=2.03$, $\mathrm{p}=0.006)$ and having more commercial male partners $(>4$ versus $\leq 4$ commercial male partners, $\mathrm{AOR}=1.49, \mathrm{p}=$ $0.053)$ reported a higher rate of HIV testing. Condom use was not associated with HIV testing among MBs. Among other MSM, HIV testing was reported more often for those with homosexual orientation than for those with bisexual orientation (58\% versus $37 \%, \mathrm{p}=0.003)$ and for those with heterosexual orientation ( $58 \%$ versus $28 \%, \mathrm{p}=0.018)$. Other MSM who had only male sex partners $(\mathrm{AOR}=1.82$, $\mathrm{p}=0.026)$, and those having a STI history $(\mathrm{AOR}=2.53$, $\mathrm{p}=0.004)$ reported more often HIV testing. Living in

Erasmus MC, University Medical Center Rotterdam, Rotterdam, the Netherlands 